\title{
Qualidade de vida e qualidade do sono na percepção de residentes multiprofissionais: estudo transversal
}

\section{Quality of life and quality of sleep in multiprofessional residents perception: a cross-sectional study}

\author{
Fernanda Bastos ${ }^{1}$, Maria Eduarda Lara de Oliveira ${ }^{1}$, Eduarda Chaves Silveira ${ }^{1}$, Dulciane Nunes \\ Paiva $^{1}$, Éboni Marilia Reuter ${ }^{1}$
}

1 - Universidade de Santa Cruz do Sul - UNISC, Santa Cruz do Sul, RS, Brasil.

\begin{abstract}
RESUMO
Introdução: o Programa de Residência Multiprofissional de Saúde (PRMS) é uma modalidade de pós-graduação que permite aos profissionais da área da saúde uma formação com qualidade e supervisionada, com duração de dois anos e reconhecida como uma forma eficaz. Entretanto, com seus malefícios pelo desgaste físico, emocional e a competitividade, assim podendo causar distúrbios do sono.

lara2@mx2.unisc.br Objetivo: avaliar a percepção da qualidade de vida e qualidade de sono dos Residentes de equipe multiprofissional de um hospital do interior do Sul do Brasil. Método: estudo transversal, realizado com Residentes multiprofissionais de um programa vinculado a um hospital universitário. Foram entregues instrumentos autoaplicáveis, contendo ficha de identificação e características do exercício profissional, questionário World Health Organization Quality of Life, versão bref (WHOQOL-bref) e o Índice de Qualidade de Sono Pittsburgh (PSQI). As diferenças entre os desfechos para o tempo em exercício profissional calculadas pelo teste de qui-quadrado de Pearson ou U de Mann-Whitney. Resultados: em relação à Qualidade de Vida dos 18 Residentes respondentes, baixa qualidade de vida foi predominante em todos os domínios, exceto para relações sociais, em que a maior frequência foi observada

Palavras-chave. para escore $>70 \%$. Residentes com até 24 meses de exercício profissional apresentaram maior escore Qualidade de vida: Sono; no domínio relações sociais, quando comparado aos seus pares com mais tempo de atuação. Já na qualidade de sono, foi identificado que $61 \%$ dos Residentes foram categorizados com qualidade de sono 'Ruim', embora tenham identificado uma 'Boa' qualidade de sono no último mês. Residentes com até Hospital de ensino; Equipe multiprofissional; Trabalhador da saúde. 24 meses de exercício profissional apresentaram maior pontuação no domínio relações sociais, quando comparado aos seus pares com mais tempo de atuação. Conclusão: os Residentes possuem baixos escores nos domínios de qualidade de vida, sendo que o domínio relações sociais foi diferente conforme o tempo em exercício profissional, bem como uma qualidade de sono ruim.
\end{abstract}

\section{ABSTRACT}

Introduction: the Multiprofessional Health Residency Program is a graduate program that allows healthcare professionals to have quality and supervised training, lasting two years and recognized as an effective way. However, with its harmful effects due to physical, emotional and competitiveness, it can cause sleep disorders. Objective: to evaluate the quality of life and quality of sleep perception in Residents of multiprofessional team of a South of Brazil hospital. Method: cross-sectional study carried out with multiprofessional Residents of a program linked to a university hospital. Self-administered instruments were issued, containing identification form and characteristics of professional practice, World Health Organization Quality of Life questionnaire, bref version (WHOQOL-bref), and the Pittsburgh Sleep Quality Index (PSQI). The differences between the outcomes for time in profes-

Keywords: sional practice calculated using Pearson's chi-square test or Mann-Whitney U test. Results: about the Quality of Life of the 18 respondents, low quality of life was prevalent in all domains, except for social Teaching hospital. Multi- relationships, where the highest frequency was observed for a score $>70 \%$. Residents with up to 24 professional team; Health their peers with longer experience. As for sleep quality, it was identified that 10 of the Residents were worker. categorized as 'poor' sleep quality, although they identified a 'good' sleep quality in the last month. Conclusion: Residents have low scores in the quality of life domains, and the social relation's domain was different according to the time in professional practice, as well as poor sleep quality. 


\section{INTRODUÇÃO}

O Programa de Residência Multiprofissional em Saúde (PRMS) é uma modalidade de pósgraduação classificada como lato sensu, ${ }^{1}$ que tem como foco a educação em serviço, tendo como finalidade possibilitar uma formação de qualidade aos profissionais da área da saúde, através de acompanhamento supervisionado, fazendo com que os residentes consigam lidar com a dualidade de serem estudantes de pós-graduação, mas também profissionais habilitados por seus respectivos conselhos profissionais. ${ }^{2}$ Os programas têm dois anos de duração com carga horária total de 5760 horas, tendo $80 \%$ da carga horária de atividades práticas e $20 \%$ de atividades teóricas ou teórico-práticas totalizando, 60 horas semanais, que priorizam as atividades em atenção primária à saúde e hospitalares. ${ }^{3}$

A Residência é reconhecida como uma forma eficiente para a qualificação dos profissionais, entretanto pode gerar desgaste físico e mental, proveniente das condições laborativas, sobrecarga de trabalho e baixa remuneração. Tais fatores podem incluir o medo de fracassar, de ser mal interpretado, longas jornadas, cansaço emocional e físico, competitividade no ambiente de trabalho, não ter reconhecimento no trabalho realizado, atividades estafantes e insegurança. ${ }^{4,5}$

A qualidade de vida abrange dimensões físicas, psicológicas, tecnológicas e sociais, correspondendo a uma organização mais saudável e humana. Sendo este fato importante para a realização de qualquer atividade, que tem como enfoque a melhoria da qualidade de vida da população, sendo assim, é fundamental que os profissionais tenham plenas condições de trabalho e de vida. ${ }^{6}$

Nesse contexto, os distúrbios do sono são frequentes, podendo refletir no aumento da morbidade e do risco da ocorrência de acidentes, redução do desempenho, instabilidade de humor, diminuição da produtividade, menor expectativa de vida, transtornos do relacionamento social e comportamento, reduzindo a concentração e atenção e podendo causar sonolência e déficit de atenção. ${ }^{7}$

Dessa forma, o objetivo do estudo foi avaliar a percepção da qualidade de vida e qualidade de sono dos residentes de equipe multiprofissional de um hospital do interior do Sul do Brasil.

\section{MÉTODO}

Estudo transversal, conduzido em um hospital considerado o principal centro de saúde do Vale do Rio Pardo. Foram convidados a participar os Residentes do PRMS, na área de concentração em Atenção em Urgência e Emergência, que aceitaram participar desta pesquisa através da assinatura do Termo de Consentimento Livre e Esclarecido. Foram excluídos os residentes em período de férias ou em período de intercâmbio. O ministério da Saúde concedeu 20 bolsas anuais, para as áreas de Fisioterapia, Enfermagem, Farmácia, Psicologia, Nutrição, Odontologia, Educação Física e Serviço Social.

Para realização da coleta de dados foi entregue uma ficha de identificação na qual consta os dados como idade, sexo, naturalidade, cidade atual, formação, tempo em exercício profissional e em qual ciclo da residência se encontrava. $O$ tempo de profissão foi considerado para análises comparativas, sendo definidas as categorias: $\leq 24$ meses e $>24$ meses. Juntamente foi entregue o questionário World Health Organization Quality of Life, versão bref (WHOQOL-bref), e o instrumento Índice de qualidade de sono Pittsburgh (PSQI). Foi realizada a entrega dos instrumentos em outubro/2018 para os Residentes em que todos os questionários eram autoaplicados, sendo possibilitado a entrega dos instrumentos em uma segunda oportunidade (até 15 dias após). Ressaltamos que a presente pesquisa foi devidamente aprovada pelo Comitê de Ética em Pesquisa da Universidade de Santa Cruz do Sul (n 2.902.219).

\section{World Health Organization Quality of Life-bref}

Foi entregue o questionário WHOQOL-bref, versão em português, ${ }^{8}$ composto por 26 questões que avaliam quatro aspectos da qualidade de vida, sendo duas questões gerais (questão 1 e 2) e as 24 restantes abordam os domínios: físico (questões $3,4,10,15$, $16,17,18)$, psicológico (questões 5, 6, 7, 11, 19, 26), relações sociais (questões $21,22,23$ ) e meio ambiente (questões 8, 9, 12, 13, 14, 23, 24, 25). As respostas seguem a escala do tipo Likert (pontuam de 1 a 5 , quanto maior a pontuação maior a qualidade de vida). Para este instrumento é necessário recodificar o valor de três questões $(3,4$ e 26) pois se tratam de itens invertidos. Para o cálculo cada um dos domínios, foram seguidas as recomedações do manual, com resultados apresentados em escores transformados de 0-100. ${ }^{9}$ Para definição de ponto de corte, foi utilizado 
como referência $70 \%$ do escore máximo, sendo que valores abaixo indicam baixa qualidade de vida. ${ }^{10}$

\section{Índice de qualidade de sono Pittsburgh}

O PSQI possibilita avaliar os padrões do sono e quantificar a sua qualidade, sendo composto por perguntas relativas às características de sono durante os últimos 30 dias. São 19 questões divididas em 7 categorias: qualidade e latência do sono, sua duração e eficiência habitual, transtornos do sono, uso de medicação para dormir e disfunção diurna. Cinco questões são respondidas por companheiro de quarto (quando possível), sendo que cada resposta tem um peso que varia numa escala de 0 a 3 . As pontuações são então somadas para produzirem um escore global, que varia de 0 a 21 , em que, quanto maior a pontuação, pior a qualidade do sono: entre 0 a 5 boa qualidade do sono e acima desse valor, baixa qualidade do sono. ${ }^{11}$

Os dados foram expressos em mediana (md) e intervalo interquartil (IQ), mínimo e máximo, bem como frequência e percentual, em que as análises foram processadas no Statistical Package for the Social Sciences (versão 22.0). As comparações do tempo em exercício profissional para a qualidade de vida e qualidade do sono foram calculadas pelo teste Qui-Quadrado de Pearson para variáveis categóricas e U de Mann-Whitney para variáveis contínuas.

\section{RESULTADOS}

De um total de 38 residentes elegíveis, 19 retornaram os instrumentos, sendo um excluído por não ter concluído o seu preenchimento. A amostra final foi composta por 18 participantes, os quais tinham uma mediana de idade de 25 anos (IQ: 24 - 27 anos), 16 eram mulheres. Entre as profissões, participaram as áreas: farmácia $(n=6)$, fisioterapia $(n=4)$, odontologia $(n=3)$, nutrição e psicologia $(n=2$, cada) e serviço social $(n=1)$. Quanto ao tempo de profissão, a mediana foi de 24 meses de atuação (IQ: 17,4 - 38,7), sendo 9 participantes R1 e 9 R2.

Em relação à qualidade de vida, foi identificado que o escore $<70 \%$ foi predominante nos domínios físico, psicológico e meio ambiente, enquanto que para relações sociais, a maior frequência foi observada para $>70 \%$. Todos os Residentes apresentaram escore < $70 \%$ para domínio físico, sendo o valor mínimo de 31 e máximo de 69 . Nos domínios psicológico e relações sociais o valor mínimo foi de 44 para ambos, deferindo no valor máximo, de 75 e 94, respectivamente. No domínio meio ambiente a mediana foi de 69, sendo que o IQ apresentou mínima variação (IQ: 68 - 70). Dez dos 14 sujeitos classificados com escore $<70 \%$ apresentaram o valor de 69 para este domínio (tabela $1)$.

Em análises adicionais, foi observado que os Residentes com até 24 meses em exercício profissional apresentaram maior pontuação no domínio relações sociais, quando comparado aos seus pares com mais tempo de atuação ( $\leq 24$ meses: md 81 IQ $75-94$ versus $>24$ meses: md 69 IQ $56-75 ; p=0,027$ ). Não foram observadas diferenças para os demais domínios na comparação por tempo de profissão para dados contínuos e não foram observadas diferenças

Tabela 1 - Qualidade de vida (pelo WHOQOL-Breif) de Residentes de um programa Multiprofissional em Saúde de um hospital do interior do Sul do Brasil, 2018/19.

\begin{tabular}{lc}
\hline Variáveis & $\mathrm{n}=18$ \\
\hline Domínio físico [md;IQ] & $50(44-56)$ \\
Escore $<70 \%$ & 18 \\
Escore $>70 \%$ & 0 \\
Domínio psicológico [md;IQ] & 16 \\
Escore $<70 \%$ & 2 \\
Escore $>70 \%$ & $75(66-81)$ \\
Relações sociais [md;IQ] & $63)$ \\
Escore $<70 \%$ & 12 \\
Escore $>70 \%$ & $69(68-70)$ \\
Meio ambiente [md;IQ] & 14 \\
Escore $<70 \%$ & 4 \\
Escore $>70 \%$ & 6
\end{tabular}

md: mediana; IQ: intervalo interquartil. 
para os dados categóricos.

A mediana do somatório dos componentes do PSQI foi 6 (IQ: $5-8$ ). Foi identificado durante o último mês que 10 dos Residentes foram categorizados com qualidade de sono 'Ruim', ao mesmo tempo que a percepção sobre a qualidade do sono foi predominantemente 'Boa'. Em relação à dificuldade em manter o entusiasmo na realização de atividades habituais, a maior frequência foi para 'Um problema razoável' (tabela 2).

Em relação aos motivos pelos quais os Residentes apresentaram dificuldades para dormir, foi identificado que a maior frequência foi acordar no meio da noite ou prematuramente pela manhã, com 15 participantes referindo tal situação pelo menos 1 vez na semana. Outros motivos com maior frequência incluem precisar levantar para ir ao banheiro e não conseguir adormecer em até 30 minutos (Tabela 3 ).

\section{DISCUSSÃO}

Com o objetivo de avaliar a qualidade de vida e qualidade de sono dos participantes de um PRMS de um hospital universitário do interior do Sul no Brasil, foi identificado majoritariamente participantes com escores abaixo do critério estabelecido, sendo identificado uma diferença de pontuação no domínio de relações sociais quando comparado o tempo de formação. Ainda, uma proporção notável desses profissionais apresentou qualidade de sono ruim.

Tabela 2 - Qualidade de sono (pelo Índice de qualidade de sono Pittsburgh) de Residentes de um programa Multiprofissional em Saúde de um hospital do interior do Sul do Brasil, 2018/19.

\begin{tabular}{|c|c|}
\hline Variáveis & $\mathrm{n}=18$ \\
\hline Tempo para dormir (minutos) & $20(10-40)$ \\
\hline Tempo de sono (horas) & $7,00(6,00-7,25)$ \\
\hline \multicolumn{2}{|c|}{ Qualidade do sono no último mês } \\
\hline Muito boa & 1 \\
\hline Boa & 10 \\
\hline Ruim & 7 \\
\hline Muito ruim & - \\
\hline \multicolumn{2}{|l|}{ Uso medicamento para dormir } \\
\hline Nenhuma & 16 \\
\hline Menos de $1 \mathrm{vez} / \mathrm{sem}$ & 1 \\
\hline 1 ou 2 vezes/sem & 1 \\
\hline 3 ou mais vezes $/ \mathrm{sem}$ & - \\
\hline \multicolumn{2}{|l|}{ Dificuldade de ficar acordado } \\
\hline Nenhuma & 8 \\
\hline Menos de $1 \mathrm{vez} / \mathrm{sem}$ & 3 \\
\hline 1 ou 2 vezes/sem & 6 \\
\hline 3 ou mais vezes/sem & 1 \\
\hline \multicolumn{2}{|c|}{ Dificuldade em manter o entusiasmo } \\
\hline Nenhuma dificuldade & 2 \\
\hline Um pouco leve & 2 \\
\hline Um problema razoável & 7 \\
\hline Um grande problema & 3 \\
\hline \multicolumn{2}{|l|}{ Classificação } \\
\hline Boa qualidade do sono & 8 \\
\hline Ruim qualidade do sono & 10 \\
\hline
\end{tabular}

md: mediana; IQ: intervalo interquartil. 
Tabela 3 - Situações pelas quais os Residentes de um Programa Multiprofissional em Saúde de um hospital do interior Sul do Brasil, apresentaram dificuldades para dormir (pelo Índice de qualidade de sono Pittsburgh), durante o último mês, 2018/19.

\begin{tabular}{lcccc}
\hline Situações & Nenhuma & $<1$ vez/sem & 1 ou 2 vezes/sem & $\geq 3$ vezes/sem \\
\hline Adormecer & 2 & 8 & 3 & 5 \\
Acordou & 1 & 2 & 9 & 6 \\
Banheiro & 4 & 5 & 5 & 4 \\
Respirar & 14 & 2 & 1 & 1 \\
Frio & 15 & 2 & 1 & - \\
Calor & 14 & 1 & 3 & - \\
Sonhos & 7 & 5 & 5 & 1 \\
Dor & 5 & 9 & 3 & 1 \\
\hline
\end{tabular}

A presente pesquisa revela dados de Residentes de um PRMS que possui como características ser localizado no interior do Estado, que iniciou suas atividades em 2015 e com ênfase em Urgência e Emergência. Portanto, tratando de achados inéditos dos Residentes deste programa, em um contexto de escassas publicações com essa categoria de trabalhadores. Como limitações, a taxa de respostas dos Residentes foi de $50 \%$, o que refletiu no reduzido tamanho amostral. Além disso, os participantes não foram questionados quanto a sua saúde mental, incluindo o acompanhamento em serviços de saúde mental ou tratamento medicamentoso para transtornos mentais.

No estudo em tela, as medianas estiveram majoriatriamente abaixo de $70 \%$ do escore máximo nos domínios físico, psicológico e meio ambiente, similar aos achados de estudo que avaliou 84 Residentes médicos, em Goiânia,12 os quais obtiveram médias variando entre 59,86 a 62,67 para os referidos domínios, em que estes autores destacam que a carga horária semanal relatada variava entre 40 a 100 horas, sendo que $67 \%$ deles realizavam atividades laborais além da residência. No Paraná, 136 Residentes entre o primeiro e quinto ciclo, apresentaram as seguintes médias: domínio físico de $61,7 \pm 14,7$, psicológico 60,6 $\pm 14,5$, relações sociais $65,0 \pm 18,9$ e meio ambiente $56,8 \pm 11,7$, sem diferença entre os sexos, anos de residência e entre as especialidades. ${ }^{13}$

Em estudo ${ }^{14}$ com amostra composta por 45 participantes de um PRMS de Pernambuco, e idade média de 27 anos, o desempenho por domínio no WHOQOL-Bref mostrou que o domínio relações sociais obteve a maior média, seguido do domínio psicológico, físico e meio ambiente, vindo ao encontro dos achados no presente estudo, que também evidenciou a maior pontuação no domínio relações sociais.

Residentes multiprofissionais de Uberaba $(\mathrm{MG})^{4}$ apresentaram o domínio relações sociais como menor pontuação entre os R1. Os achados do presente estudo evidenciam que o tempo de formação profissional esteve associado a este domínio, com piores indicadores acima de 24 meses de atuação profissional. Cabe salientar que foram avaliados os Residentes ao final dos ciclos, momento em que o R1 está adaptado à rotina da residência e o R2 imerso na composição do Trabalho de Conclusão e com preocupações sobre seu futuro profissional, podendo ter influenciado os achados. Ao contrário, parte da amostra de Uberaba $(\mathrm{MG})^{4}$ era ingressante da primeira semana do programa. Ainda, o item relações sociais é o domínio com menor confiabilidade no WHOQOLbref. ${ }^{15}$

No presente estudo, uma proporção expressiva dos Residentes apresentou qualidade de sono 'ruim'. Ao avaliar a qualidade de sono pelo mesmo instrumento, comparando 105 residentes médicos e 101 estudantes de graduação de medicina, os Residentes apresentaram pior qualidade de sono (média: 6,76 e DP: 2,81 versus média: 5,90 e DP 2,39). Foram destacados os valores de duração do sono e a qualidade subjetiva do sono entre os Residentes, sem que tenha ocorrido diferenças entre os demais domínios. ${ }^{7}$ Entretanto, os grupos apresentaram resultados similares para sonolência.

Entre os fatores contributivos para a dificuldade de dormir, foi identificado o despertar muitas vezes durante a noite ou acordar muito cedo. Em 184 estudantes de medicina do Acre, a prevalência de 
qualidade ruim de sono entre os alunos foi de $61,9 \%$ $(n=112)$ e 26 alunos foram incluídos no padrão de distúrbios do sono, sendo o tempo em que demoram a dormir entre 16 a 30 minutos, o que vem ao encontro dos achados do presente estudo. Entre os acadêmicos que apresentaram distúrbio do sono, relataram dormir menos de 5 horas por noite. Quanto aos fatores que interferem na qualidade do sono estavam as preocupações $(81,1 \%)$, calor $(72,1 \%)$, acordar muito cedo $(64,7 \%)$ e dores $(40,5 \%){ }^{16}$

Estudo ${ }^{17} \mathrm{com} 59$ residentes de psiquiatria refere que as categorias do PSQI com maiores escores foi a disfunção diurna $(1,37)$, distúrbios do sono $(1,22)$ e qualidade subjetiva do sono $(1,15)$. Os Residentes do terceiro ano tiveram pior qualidade de sono quando comparado aos residentes do primeiro e segundo ano. Em relação aos 35 residentes que apresentaram qualidade de sono ruim, $80 \%$ deles nunca procurou por um médico em relação ao problema do sono e $25,7 \%$ usava medicação para dormir. Deve ser considerado que nesse contexto de formação em ensino, as características laborais incluem uma carga horária semanal extensa ${ }^{18}$, bem como atribuições que permeiam demais profissionais do serviço e usuários ${ }^{19}$, os quais podem refletir na percepção da qualidade de vida e de sono dessa categoria de profissionais.

\section{CONCLUSÃO}

Foi observado que os residentes multiprofissionais de um Hospital universitário do interior do Sul do Brasil, apresentaram baixos escores para domínios do WHOQOL-bref, com diferença no domínio relações sociais quando comparado o tempo em exercício profissional, e uma qualidade do sono ruim. Desta forma, se identifica a necessidade de atenção à saúde desses profissionais, considerando que o período de residência envolve um intenso desenvolvimento pessoal e profissional, com situações desafiadoras, devido às suas responsabilidades.

\section{REFERÊNCIAS}

1. Cheade MFM, Frota OP, Loureiro MDR, Quintanilha ACF. Residência multiprofissional em saúde: a busca pela integralidade. Revista Cogitare Enfermagem 2013;18(3):592-5. doi: http://dx.doi.org/10.5380/ce.v18i3.46360

2. Ferreira ECN, Ribeiro FV, Neto RPS, Santos BN, Santo LRE, Prince KA, Oliveira MVM. Síndrome de burnout em residentes médicos de montes claros/MG. Revista Atenção em Saúde 2017;15(53):44-9. doi: https://dx.doi.org/10.13037/ras. vol15n53.4587

3. Silva JC, Contim D, Ohl RIB, Chavaglia SRR, Amaral EMS. Percepção dos residentes sobre sua atuação no programa de residência multiprofissional. Revista Acta Paulista de Enfermagem 2015, 28(2):132-8. doi: https://dx.doi. org/10.1590/1982-0194201500023

4. Moreira APF, Patrizzi LJ, Accioly MF, Shimano SGN, Walsh IAP. Avaliação da qualidade de vida, sono e Síndrome de Burnout dos residentes de um programa de residência multiprofissional em saúde. Revista Medicina Ribeirão Preto 2016;49(5):393-402. doi: https://dx.doi. org/10.11606/issn.2176-7262.v49i5p393-402

5. Silva MRA, Silveira PRRM. Estresse ocupacional em enfermeiros residentes de um programa de residência multiprofissional em saúde da família. Revista Brasileira de Inovação Tecnológica em Saúde 2017;7(1):24-35. doi: https:// dx.doi.org/10.18816/r-bits.v7i1.11681

6. Santos AA, Costa ORS. Qualidade de vida no trabalho dos profissionais de enfermagem que atuam no período noturno em um hospital escola do Sul de Minas Gerais. Revista Ciências em Saúde 2016;6(1):37-50. doi: https://dx.doi.org/10.21876/ resfmit.v6i1.453

7. Purim KSM, Guimarães ATB, Titski ACK, Leite N. Privação do sono e sonolência excessiva em médicos residentes e estudantes de medicina. Revista do colégio Brasileiro de cirurgiões 2016;43(6):438-44. doi: https://dx.doi.org/10.1590/010069912016006005

8. Fleck MPA, Louzada S, Xavier M, Chachamovich E, Vieira G, Santos L, Pinzon V. Aplicação da versão em português do instrumento abreviado de avaliação da qualidade de vida “WHOQOL- bref'. Revista de Saúde Pública 2000; 9(3):47381. doi: http://dx.doi.org/10.1590/S0034-89102000000200012

9. World Health Organization (1996) WHO-BREF: Introduction, administration, scoring and generic version of the assessment. 1996. Geneva: WHO. http://www.who.int/mental_health/media/ en/76.pdf Accessed 9 August 2017

10. Xia P, Li N, Hau K-T, Liu C, Lu Y. Quality of life of Chinese urban community residents: a psychometric study of the mainland Chinese version of the WHOQOL-BREF. BMC Med Res Methodol 2012;12:37. https://doi.org/10.1186/1471-228812-37

11. Buysse DJ, Reynolds III CF, Monk TH, Berman SR, Kupfer DJ. The Pittsburgh Sleep Quality Index: a new instrument for psychiatric practice and research. Psychiatry Res. 1989 May;28(2):193-213. https://doi.org/10.1016/01651781(89)90047-4

12. Dias BA, Pereira MN, Sousa IF, Almeida RJ. Qualidade de vida de médicos residentes de um hospital escola. Revista Scientia Medica, 2016; 26(1):89-91.

13. Asaiag PE, Perotta B, Martins MA, Tempski P. Avaliação da Qualidade de Vida, Sonolência Diurna e Burnout em Médicos Residentes. Revista Brasileira de Educação Médica 2010;34(3):422-9. doi: https://dx.doi.org/10.1590/S010055022010000300012

14. Cahú RAG, Santos ACO, Pereira RC, Vieira CJL, Gomes SA. Estresse e qualidade de vida em residência multiprofissional em saúde. Revista Brasileira de Terapias Cognitivas 2014;10(2):7683. doi: http://dx.doi.org/10.5935/1808-5687.20140013

15. Serra AV, Canavarro MC, Simões M, Pereira M, Gameiro S, Quartilho MJ, Rijo D, Carona C, Paredes T. Estudos psicométricos 
do instrumento de avaliação da qualidade de vida da Organização Mundial de Saúde (WHOQOL-Bref) para Português de Portugal.

Revista Psiquiatria Clínica 2006;27(1):41-9.

16. Ribeiro CRF, Silva YMGP, Oliveira SMC. O impacto da qualidade do sono na formação médica. Revista Sociedade Brasileira de Clinica Médica 2014;12(1):8-14.

17. Melo MCA, Medeiros FC, Bruin VMS, Santana JAP, Lima AB, Daher EF. Sleep Quality Among Psychiatry Residents. La Revue Canadienne de Psychiatrie 2016;61(1):44-9. doi: http:// dx.doi.org/10.1177/0706743715620410

18. Rezende GL, Mello MSMS, Granjeiro RC, Nakanishi M, Oliveira CAPC. A qualidade de vida entre os residentes de Otorrinolaringologia do Distrito Federal. Braz. J. Otorhinolaryngol 2011;77(4):466-72. doi: https://dx.doi. org/10.1590/S1808-86942011000400010

19. Abreu-Reis P, Oldoni C, de-Souza GAL, Bettega AL, Góes MN, Sarquis LM, Brunello LFS, Tomasich, FS, Collaço IA, Nasr A. Aspectos psicológicos e qualidade de vida na Residência Médica. Revista do Colégio Brasileiro de Cirurgiões 2019;46(1):e2050. doi: https://dx.doi.org/10.1590/01006991e-20192050

Como citar: BASTOS, Fernanda et al. Qualidade de vida e qualidade do sono na percepção de residentes multiprofissionais: estudo transversal. Revista Interdisciplinar de Promoção da Saúde, Santa Cruz do Sul, v. 3, n. 1, jan. 2020. ISSN $2595-3664$. Disponível em: <https://online.unisc.br/seer/index.php/ripsunisc/article/view/15870>. Acesso em: 01 jan. 2020. doi:https://doi. org/10.17058/rips.v3i1.15870 